\title{
Short Pulse Generation Using Multisegment Mode-Locked Semiconductor Lasers
}

\author{
Dennis J. Derickson, Member, IEEE, Roger J. Helkey, Member, IEEE, Alan Mar, \\ Judy R. Karin, Student Member, IEEE, John G. Wasserbauer, Student Member, IEEE, \\ and John E. Bowers, Senior Member, IEEE \\ Invited Paper
}

\begin{abstract}
Mode-locked semiconductor lasers which incorporate multiple contacting segments are found to give improved performance over single-segment designs. The functions of gain, saturable absorption, gain modulation, repetition rate tuning, wavelength tuning, and electrical pulse generation can be integrated on a single semiconductor chip. The optimization of the performance of multisegment mode-locked lasers in terms of material parameters, waveguiding parameters, electrical parasitics, and segment length is discussed experimentally and theoretically.
\end{abstract}

\section{INTRODUCTION}

QEMICONDUCTOR lasers are important sources of short optical and electrical pulses. Semiconductor lasers are small in size, use electrical pumping, are easy to operate, and consequently short optical pulses can now be used in applications that were previously infeasible or uneconomical. These pulses are used in high-speed optical fiber communication systems using time-division multiplexing for transmitters and for demultiplexing at the receiver [1], [2]. Short optical pulses are also being used for optoelectronic measurement applications such as electrooptic sampling [3], analog to digital (A-D) conversion [4], and impulse response testing of optical components.

This paper concentrates on advances in the short pulse generation field that are a result of using multiple-segment semiconductor laser structures. Three such structures that will be studied are shown in Fig. 1. Fig. 1(a) and (b) shows monolithic and external-cavity mode-locked semiconductor laser configurations. Fig. 1(c) shows a structure which can be used to generate optical pulses by gainswitching or $Q$-switching techniques. Multisegment mode-locked lasers have produced very short single optical pulsewidths, and allow device configurations which were previously not obtainable.

Section II describes the performance of the individual structures shown in Fig. 2. Section III gives a comprehensive comparison of the important characteristics such as pulsewidth, spectral width, amplitude noise, phase

Manuscript received February 28, 1992; revised April 11, 1992. This work was supported by the Office of Naval Research. The work of R. J. Helkey was supported by a Newport Research Fellowship.

The authors are with the Department of Electrical and Computer Engineering, University of California, Santa Barbara, CA 93106

IEEE Log Number 9202629.

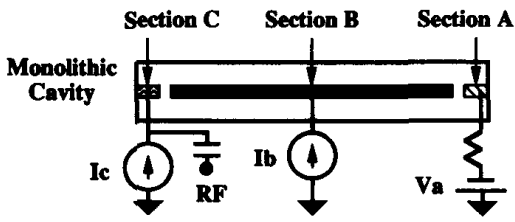

(a)

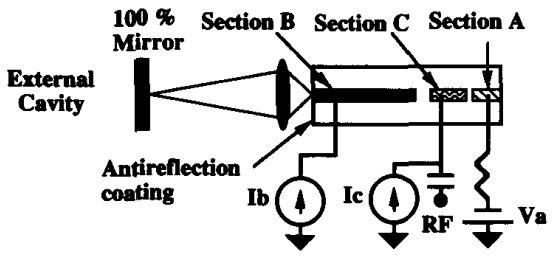

(b)

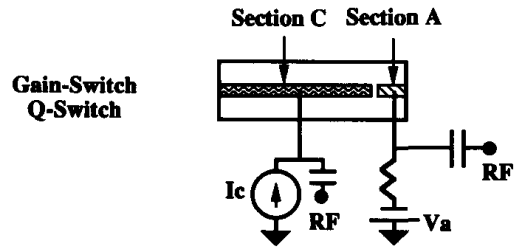

(c)

Fig. 1. Multisegment structures for generating short optical and electrical pulses. (a) Monolithic cavity mode-locked semiconductor laser. (b) External cavity mode-locked semiconductor laser. (c) Gain-switched or $Q$-switched laser. Section A is the waveguide saturable absorber, Section $\mathrm{B}$ is the gain section, and Section $\mathrm{C}$ is the gain modulation segment.

noise, pulse energy, and repetition rate of these devices. Section IV addresses the optimization of multisegment designs in terms of the free parameters in the design such as segment length, active region parameters, electrical parasitics, and electrical drive signals. Section V discusses the limitations on achievable pulsewidth for these structures. Finally, Section VI gives a discussion on future directions for the field and conclusions of this paper.

\section{Multiple-Segment Structures}

This section describes the importance of multisegment structures for short pulse generation and compares the performance of the three device types shown in Fig. 1. There are six different mode-locking configurations that will be discussed. Active, passive, and hybrid (activepassive) modulation techniques will be considered for both 
(a)
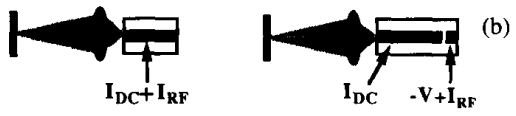

(c)
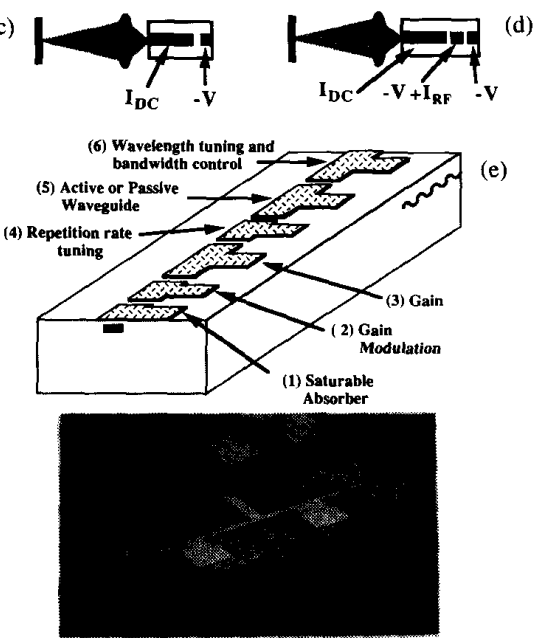

(f)

Fig. 2. Multisegment mode-locked semiconductor laser configurations. (a) Single-segment external cavity active mode locking. (b) Two-segment external cavity active mode locking. (c) Two-segment external cavity passive mode locking. (d) Three-segment external cavity hybrid mode locking. (e) Functionality in a monolithic cavity mode-locked laser structure. (f) Scanning electron micrograph of a multiple-segment mode-locked laser structure.

monolithic [Fig. 1(a)] and external cavity [Fig. 1(b)] configurations. The mode-locking results will be contrasted with gain-switching and $Q$-switching measurements on two-segment lasers [Fig. 1(c)].

\section{A. Actively Mode-Locked External-Cavity Lasers}

The simplest external-cavity configuration for mode locking is the single-segment design of Fig. 2(a). It consists of a semiconductor diode laser with a single pumping electrode and an antireflection coated facet coupled to an external cavity. The external cavity contains a collimating lens, a feedback mirror, and possibly a wavelength control filter. In active mode locking, the gain of the laser is modulated with electrical pulses that have a period nearly equal to the round-trip time in the laser cavity. In order to get very short optical pulses from the device, the electrical modulation pulse must be narrow and of large amplitude in order to create a very short time window of net gain in the device. The laser must also have a small parasitic capacitance and small resistance so that the highfrequency components of the electrical pulse are not attenuated. Pulses as short as $0.6 \mathrm{ps}$ with $0.024 \mathrm{pJ}$ of energy have been generated using active mode locking of singlesegment semiconductor lasers [5]. The device was actively mode locked with a $27 \mathrm{dBm}$ sinusoid at a $16 \mathrm{GHz}$ repetition rate. The problem with a single segment device is that it is difficult to get short single pulse outputs from the laser. Secondary pulses spaced at the round-trip time in the laser diode are often generated. These secondary pulses are initiated from imperfect antireflection coatings on the laser diode facet. Over many round-trips, the secondary pulses are amplified to energy values that are significant compared to the main pulse. Simulations have shown that power reflection coefficients as small as $10^{-5}$ can cause multiple pulse formation [6]. In order to suppress the formation of secondary pulses, the net gain for the secondary pulse should be much less than unity so that there is no amplification of the secondary pulse. For effective suppression, the electrical modulation pulsewidth should be as narrow as possible. High frequency sinusoidal signals (e.g., $>5 \mathrm{GHz}$ ) provide narrow effective pulsewidths, but for many applications lower repetition rates are required. Step recovery diodes pulses can be used at lower repetition rates (e.g., $<5 \mathrm{GHz}$ ). The round-trip time in the laser diode should be long enough so that the electrical modulation pulse is not pumping the gain region during the transit of the secondary pulse through the laser diode. It is also desirable for the electrical modulation signal to remove carriers from the active region during the off period of the modulation.

The functions of gain and gain modulation are accomplished in the same segment in the device of Fig. 2(a). By separating the gain and gain modulation functions into two separate locations as in Fig. 2(b), the multiple pulsation problem can be suppressed. The long segment is forward biased with a dc current to provide the over-all gain for the device. The short segment is reverse biased and driven by the electrical modulation pulse. In order to achieve a lower repetition rate, a step recovery diode with 29 ps pulsewidth and $7 \mathrm{~V}$ amplitude (into a $50 \Omega$ load) was used as a modulation source at $3 \mathrm{GHz}$. Since the modulation segment is reverse biased, only the peak of the modulation cycle actually injects charge into the short segment resulting in an even shorter effective pumping interval. After the electrical modulation pulse ends, the reversebiased segment removes carriers from the active region of the short segment, resulting in a very high loss condition for secondary pulses. Fig. 3 shows autocorrelation results for two-segment actively mode-locked semiconductor lasers with laser diode lengths of $450 \mu \mathrm{m}$ [Fig. 3(a)] and $1000 \mu \mathrm{m}$ [Fig. 3(b)]. For the $450 \mu \mathrm{m}$ experiment, the round-trip time of $10 \mathrm{ps}$ in the laser diode is not sufficient for the modulation pulse to decay and allow the short segment to recover to a high loss condition for the arrival of the reflected pulses. When the laser length is increased to $1000 \mu \mathrm{m}$, the round-trip time is increased to $22 \mathrm{ps}$ giving sufficient time for the reverse bias to aid in recovering the short segment to a high loss condition to prevent multiple pulse formation. Single pulses as short as $1.4 \mathrm{ps}$ [7] and energies as high as $3.5 \mathrm{pJ}$ [8] have been achieved using this two-section active mode-locking technique.

\section{B. Passively and Hybridly Mode-Locked External- Cavity Lasers}

Passive mode-locking techniques are used in many laser systems to generate short optical pulses. The key component necessary for passive mode locking is a suitable 


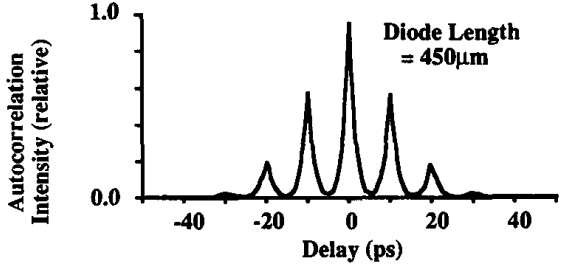

(a)

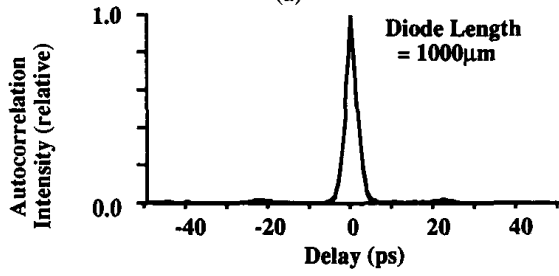

(b)

Fig. 3. Autocorrelation traces for the two-segment mode-locked laser of Fig. 2(b) for a cavity length of (a) $450 \mu \mathrm{m}$ and (b) $1000 \mu \mathrm{m}$.

saturable absorber. The saturable absorbers used in semiconductor laser systems are slow saturable absorbers [9]. This means that the leading edge of an optical pulse is absorbed and the trailing edge of the optical pulse is unattenuated during the passage of the optical pulse through the saturable absorber. Slow saturable absorption in conjunction with gain saturation during the trailing edge of the optical pulse leads to a net pulse narrowing on each transit around the mode-locked laser cavity. For successful passive mode locking, the absorption of the saturable absorber must saturate at a lower energy than the gain of the gain medium. The saturation energy of a material is defined as

$$
E_{\mathrm{sat}}=\frac{h \nu A}{\frac{\partial g}{\partial N}}
$$

where $h$ is Planck's constant, $\nu$ is the optical frequency, $A$ is the mode cross-sectional area, and $\partial g / \partial N$ is the differential gain with respect to carrier density. The saturation energy is a measure of the energy necessary to saturate the gain of a gain segment or the absorption of an absorber segment.

Suitable absorbers can be made with semiconductor laser materials. The slope of the gain versus carrier density function in semiconductor lasers decreases in value as the carrier density level is increased [10]. Since the carrier density level in saturable absorbers is much smaller than in gain regions, semiconductor saturable absorbers will have lower saturation energies than semiconductor gain regions. The reduction in differential gain with increased carrier density can be particularly large in quantum-well lasers due to the step-like density-of-states function in quantum wells. The mode cross-sectional area can also be useful in reducing the saturation energy of saturable absorbers but it is difficult to achieve a mode crosssectional area that is much smaller than the already tightly confined mode found in edge-emitting lasers. Reduction of the mode cross-section area in a saturable absorber should be an effective technique in vertical-cavity surface-emitting lasers in which the gain region may have a mode diameter of over $10 \mu \mathrm{m}$ [11].

The saturable absorber must quickly recover to the large absorption state after the passage of the optical pulse for passive mode locking to be stable with a slow saturable absorber [9]. Fast saturable absorber recovery is also important for achieving high repetition rates since the $a b-$ sorber must reset to a large attenuation value on each round-trip. The absorption recovery time constant due to spontaneous emission recombination, typically $1 \mathrm{~ns}$, is too long for effective absorption recovery. This has led researchers to introduce damage to the material in order to decrease the nonradiative lifetime of the material. Proton and nitrogen bombardment of laser diode facets have been successful in producing mode-locking results [12], [13]. There is some question as to the long-term reliability of the damage centers created with these techniques. External-cavity saturable absorbers which use the excitonic absorption feature of multiquantum-well samples have also been implemented [14]. A more convenient method for achieving passive mode locking is with the incorporation of waveguide saturable absorbers as shown in the twosection laser of Fig. 2(c) [15]. The long laser section is forward biased to provide gain for the laser. By reverse biasing the short segment of the laser, a waveguide saturable absorber is formed. Reverse-biased laser segments also behave as waveguide photodetectors. The incoming optical pulse saturates the band-to-band absorption (excitonic absorption is not the dominant process) and bleaches the short segment. After the passage of the optical pulse through the absorber section, the electric field in the photodetector sweeps the carriers out of the active region and returns the segment to the high attenuation state. Single pulses as short as $2.5 \mathrm{ps}$ with $0.7 \mathrm{pJ}$ of energy have been achieved in these two-section passively mode-locked structures [16]. Similar pulsewidths were obtained over a wide repetition rate from $0.2-9 \mathrm{GHz}$ with a similar pulse energy independent of repetition rate.

The absorption recovery time of waveguide saturable absorbers was measured using pump-probe techniques [17]. Waveguide saturable absorbers with GaAs bulk and quantum well active regions were pumped with 80 fs pulses from a mode-locked $\mathrm{Ti}$ : sapphire laser. The wavelength of the pump signal was chosen to be the same as that which would have occurred in an actual mode-locked semiconductor laser experiment. The pump pulse bleaches the absorption of the saturable absorber and a delayed probe pulse is used to measure the transmission through the absorber versus the time difference between the pump and probe signals. Fig. 4 shows a result from the pumpprobe measurements. The absorption recovers back to its maximum value in a time of less than approximately 15 ps. This fast recovery time has several important implications for mode-locked semiconductor lasers. Since the absorption recovery time sets an upper limit on the achievable repetition rate in a passively mode-locked 


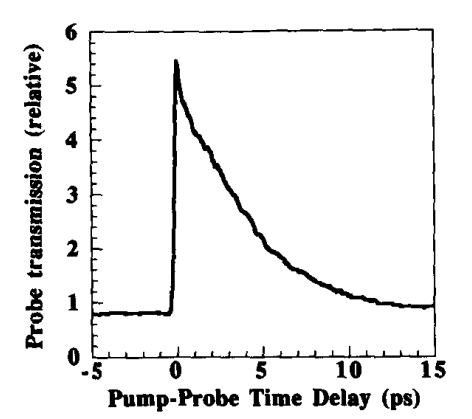

Fig. 4. Relative transmission versus pump-to-probe time delay for an 80 $\mu \mathrm{m}$ long reverse-biased waveguide saturable absorber with $4 \mathrm{GaAs}$ quantum wells.

semiconductor laser, the measured recovery time implies that very high frequency mode locking (e.g., $100 \mathrm{GHz}$ ) is possible. Experimentally, semiconductor lasers have been passively mode locked with repetition rates as high as $10 \mathrm{GHz}$ in external cavity lasers, and up to $350 \mathrm{GHz}$ in monolithic cavity mode-locked lasers [18]. The very fast recovery time of waveguide saturable absorbers can be used to suppress multiple pulse formation induced by imperfect antireflection coatings [16].

Since saturable absorbers act as photodetectors, electrical pulses are also generated when the optical pulse passes through the saturable absorber. A photocurrent response from a passively mode-locked semiconductor laser [16] is shown in Fig. 5. The photocurrent response is limited by the $R C$ time constant of the saturable absorber capacitance and the $50 \Omega$ resistance of the measuring sampling oscilloscope. As the carriers are swept out of the active region, a field is built up which opposes the internal photodetector field resulting in an increased carrier sweepout time. Note that the photocurrent response time is much longer than the absorption recovery time which is measured by the pump-probe experiment. This result shows that it is the transit-time response of the saturable photodetector which is important in absorption recovery. The capacitance of the saturable absorber stores the swept-out charge until it can be dissipated in a charge-bleeding resistance. It is important to discharge the capacitor on each round-trip of the mode-locked laser. The optimal termination resistance for the absorber segment should have a small value to quickly drain the capacitor's stored charge. The electrical photocurrent pulses from the saturable absorber are a useful electronic pulse source [19]. The electrical pulses can be used to synchronize the optical pulse to an electronic system. Passively mode-locked semiconductor lasers can also be used as microwave oscillators with only dc power supplies required.

Hybrid mode locking can be achieved with the addition of a separate gain modulation segment to the laser to form the three-section laser of Fig. 2(d). The requirements on the electrical modulation waveform are reduced in hybrid mode locking. Since saturable absorption is the dominant mechanism for achieving very short pulses, it is not as important to have an extremely short electrical modula-

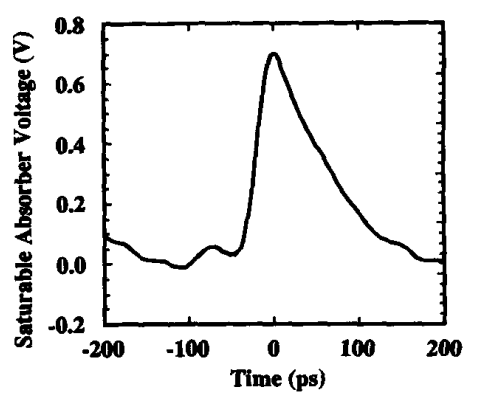

Fig. 5. Saturable absorber voltage in a $50 \Omega$ load (ac coupled measurement) due to the photocurrent from a $16 \mu \mathrm{m}$ long waveguide saturable absorber from the passively mode-locked laser structure of Fig. 2(c).

tion waveform. A larger capacitance on the modulation segment can be tolerated resulting in simpler device fabrication. Since the saturable absorber is dc reverse biased, the absorber can recover to an absorbing state only a few picoseconds after the passage of the optical pulse leading to an effective multiple pulse suppression mechanism. A three-section hybridly mode-locked laser with the configuration of Fig. 2(d) was tested. The laser diode had a GaAs bulk active region [20] and an over-all laser diode length of $500 \mu \mathrm{m}$. The modulation segment and gain segment lengths are $16 \mu \mathrm{m}$. The autocorrelation width was 2.85 ps which deconvolves to a 1.9 ps pulsewidth assuming a hyperbolic secant squared pulse shape. The spectral width was $1 \mathrm{THz}$, the pulse energy was $0.18 \mathrm{pJ}$ and the repetition rate was $6 \mathrm{GHz}$.

\section{Monolithic Cavity Mode-Locked Semiconductor Lasers}

The previous discussion of multisegment external-cavity mode-locked lasers has shown that all of the necessary functions for active, passive and hybrid mode-locked lasers can be implemented together on a single semiconductor chip. For the next step, the entire external cavity can be replaced with a semiconductor waveguide resulting in an integrated monolithic cavity mode-locked laser structure such as that shown in Fig. 2(e). Monolithic cavity devices are very small and do not have the mechanical instabilities associated with the optical elements in an external cavity. Fig. 2(f) shows a scanning electron micrograph of a monolithic cavity structure. Fig. 2(e) illustrates the wide range of functionality that is possible from a process which incorporates separated top contacts for nonuniform electrical biasing and a continuous waveguide along the entire device length. In this simple process, six distinct functions can be performed.

1) Section 1 is reverse biased to act as a waveguide saturable absorber-photodetector for passive and hybrid mode locking.

2) Section 2 is the gain modulation segment.

3) Section 3 is forward biased and provides the overall gain necessary for lasing. 
4) Section 4 is a segment that allows for electrical tuning of the repetition rate during passive mode locking by adjusting the carrier density.

5) Section 5 is the active or passive waveguide section which provides the delay necessary for achieving a specific repetition rate. Active waveguides are the easiest to fabricate in a laser process. Low-loss passive waveguides can be integrated with a more complex processing sequence [20], [21]. Multiple pulsation effects can be a problem if there is a reflection at the active-to-passive waveguide transition. Reflection problems caused by imperfect active-passive transitions can be suppressed using the multisegment external-cavity laser techniques discussed earlier.

6) Section 6 incorporates Bragg wavelength filters to control the center wavelength and bandwidth of the laser [21]. Control of the laser bandwidth is very important for optical fiber communication system applications.

Monolithic cavity designs have been demonstrated with over-all lengths between 7 and $0.25 \mathrm{~mm}$ corresponding to a repetition rate as low as $5.5 \mathrm{GHz}$ [22] and as high as $350 \mathrm{GHz}$ [18]. Such high repetition rates in the millimeter wave range are made possible by the very fast absorption recovery times of waveguide saturable absorbers. A threesegment monolithic cavity mode-locked semiconductor laser with a device length of $2 \mathrm{~mm}$ and a corresponding repetition rate of $21 \mathrm{GHz}$ was fabricated and tested. The device had 4 InGaAs quantum wells [23] and a saturable absorber length of $40 \mu \mathrm{m}$. The device was hybridly modelocked with a $24 \mathrm{dBm}$ sinusoidal signal. The resulting op-

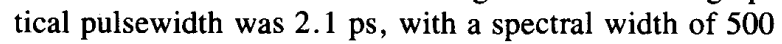
$\mathrm{GHz}$, and pulse energy of $0.04 \mathrm{pJ}$.

\section{Multisegment Gain-Switched and Q-Switched Lasers}

Gain-switched lasers are an attractive alternative to mode-locked semiconductor lasers in several aspects. The devices are small in size, don't require external cavities, and are easy to operate. The repetition rate of the device is not constrained to the round trip time in the laser. However, gain-switched lasers tend to have wider pulsewidths and larger time-bandwidth products than mode-locked lasers. The performance of single-section gain-switched lasers can be enhanced with the use of the two-segment designs shown in Fig. 1(c). Two-segment lasers can be gain switched by reverse biasing the short segment to act as a saturable absorber and applying a short electrical pulse to the main segment. In single-segment gain-switching experiments, wide pulses (e.g., $30 \mathrm{ps)}$ ) are often produced because the optical pulse is emitted early with respect to the peak of the electrical pump waveform. The presence of the saturable absorber delays the pulse emission since the saturable absorber must first be bleached. This delay of the output pulse with respect to the electrical modulation pulse causes a higher level of inversion and decreases the width of the gain-switched pulse.
In $Q$-switching experiments, the long segment is forward biased to produce gain in the device. The short segment is reverse biased to suppress lasing in the structure. When an electrical pulse is applied to the short segment, the peak of the pulse forward biases the segment resulting in a narrow time window of low loss in the cavity for the optical pulse being emitted. In both cases, it is important to have very short, large amplitude electrical modulation pulses. It is also important to have a low electrical parasitic laser process so that the high frequency components of the electrical pulse are not attenuated in the laser. Twosegment gain-switched and $Q$-switched lasers were fabricated and tested. The laser diode had a GaAs bulk active region [20] with an overall length of $150 \mu \mathrm{m}$ and a short segment length of $16 \mu \mathrm{m}$. The electrical modulation pulse was from a step-recovery diode operating at $1 \mathrm{GHz}$ with a 60 ps pulsewidth and $6 \mathrm{~V}$ amplitude into a $50 \Omega$ load. It was found that the two-segment lasers produced the shortest pulsewidths near the frequency at which they tended to self-pulsate when no modulation was applied (typically a few $\mathrm{GHz}$ ). The resulting pulsewidths for the gainswitched and $Q$-switched lasers were 13 and $15 \mathrm{ps,} \mathrm{re-}$ spectively. The pulse energy for the gain-switched device was $3.4 \mathrm{pJ}$ and the gain-switched device was $4.0 \mathrm{pJ}$. The spectral widths for the gain-switching and $Q$-switching experiments were 4 and $2.4 \mathrm{THz}$, respectively.

\section{Comparison of Device Performance}

Table I lists a comparison of the performance for the multiple-segment devices shown in Fig. 1. The pulsewidth, spectral width, time-bandwidth product, pulse energy, repetition rate, and active region composition are included in the comparison. From the results listed in the table along with additional experiments which are not included in the table, there are several important trends which can be identified.

\section{A. Pulsewidth}

The pulsewidths that have been demonstrated for multisegment external cavity mode-locked semiconductor lasers with single pulse outputs are typically in the 1-3 ps range for the active, passive, or hybrid mode-locking techniques. It is in general easier to get short optical pulses using hybrid and passive mode-locking techniques. The saturable absorber is primarily responsible for pulse narrowing with the active modulation providing amplitude and phase stabilization. Monolithic cavity mode-locked semiconductor lasers showed wider pulsewidths as the repetition rates were decreased (e.g., $5.5 \mathrm{GHz}$ ). The poorer performance of the monolithic cavity devices at the lower repetition rates is believed to be a result of the use of all-active waveguides. Gain dispersion effects [24] tend to broaden the pulses more effectively when the optical pulse propagates through long sections of active waveguide. Performance at lower repetition rates should improve with the incorporation of low-loss passive wave- 
TABLE I

Comparison of Multisegment Structure Performance

\begin{tabular}{|c|c|c|c|c|c|c|c|c|c|}
\hline $\begin{array}{l}\text { Cavity } \\
\text { Type }\end{array}$ & $\begin{array}{l}\text { Modulation } \\
\text { Technique }\end{array}$ & $\begin{array}{l}\text { Pulse width } \\
\text { (ps) }\end{array}$ & $\begin{array}{l}\text { Spectral } \\
\text { Width } \\
(\mathrm{GHz})\end{array}$ & $\begin{array}{l}\text { Time- } \\
\text { Bandwidth } \\
\text { Product }\end{array}$ & $\begin{array}{c}\text { Pulse } \\
\text { Energy } \\
(\mathrm{pJ})\end{array}$ & $\begin{array}{l}\text { Repetition } \\
\text { Rate } \\
(\mathrm{GHz})\end{array}$ & $\begin{array}{l}\text { Wavelength } \\
\qquad(\mu \mathrm{m})\end{array}$ & $\begin{array}{l}\text { Active } \\
\text { Region }\end{array}$ & Reference \\
\hline Ext. & $\begin{array}{l}\text { Active } \\
\text { Two-Seg. }\end{array}$ & 1.4 & 342 & 0.48 & 0.28 & 3 & 1.3 & Bulk & [7] \\
\hline Ext. & $\begin{array}{l}\text { Passive } \\
\text { Two-Seg. }\end{array}$ & 2.5 & 720 & 1.8 & 0.7 & 5 & 0.84 & $4 \mathrm{QW}$ & {$[22]$} \\
\hline Ext. & $\begin{array}{l}\text { Hybrid } \\
\text { Two-Seg. }\end{array}$ & 2.5 & 1000 & 2.5 & 0.8 & 5 & 0.84 & $4 \mathrm{QW}$ & [22] \\
\hline Ext. & $\begin{array}{l}\text { Hybrid } \\
\text { Three-Seg. }\end{array}$ & 1.9 & 900 & 1.71 & 0.18 & 6 & 0.83 & Bulk & {$[16],[27]$} \\
\hline Mon. & $\begin{array}{l}\text { Active } \\
\text { Two-Seg. }\end{array}$ & 13 & 330 & 4.3 & 0.19 & 5.5 & 0.84 & $4 \mathrm{QW}$ & {$[22]$} \\
\hline Mon. & $\begin{array}{l}\text { Hybrid } \\
\text { Three-Seg, }\end{array}$ & 6.5 & 540 & 3.5 & 0.18 & 5.5 & 0.84 & $4 \mathrm{QW}$ & [22] \\
\hline Mon. & $\begin{array}{l}\text { Passive } \\
\text { Two-Seg. }\end{array}$ & 10 & 400 & 4.0 & 0.25 & 5.5 & 0.84 & $4 \mathrm{QW}$ & {$[22]$} \\
\hline Mon. & $\begin{array}{l}\text { Passive } \\
\text { Two-Seg. }\end{array}$ & 5.5 & 550 & 3.0 & 0.53 & 11 & 0.84 & $4 \mathrm{QW}$ & \\
\hline Mon. & $\begin{array}{l}\text { Hybrid } \\
\text { Three-Seg. }\end{array}$ & 2.2 & 500 & 1.1 & 0.03 & 21 & 1.58 & $4 \mathrm{QW}$ & \\
\hline Mon. & $\begin{array}{l}\text { Passive } \\
\text { Two-Seg. }\end{array}$ & 1.3 & 600 & 0.78 & 0.02 & 41 & 1.58 & $4 \mathrm{QW}$ & [38] \\
\hline Mon. & $\begin{array}{c}Q \text {-Switch } \\
\text { Two-Seg. }\end{array}$ & 15 & 2400 & 36 & 4 & 1 & 0.825 & Bulk & \\
\hline Mon. & $\begin{array}{c}\text { Gain-Switch } \\
\text { Two-Seg. }\end{array}$ & 13 & 4000 & 52 & 3.4 & 1 & 0.822 & Bulk & \\
\hline
\end{tabular}

guide segments which act as a more ideal medium for short pulse propagation [21]. Two-segment gain-switched and $Q$-switched lasers generally had pulsewidths in the 10-20 ps range. Single section gain-switched lasers from the same material gave pulsewidths in the $20-30$ ps range.

\section{B. Spectral Width}

The experimental results show that for designs in which bandwidth control filter elements are not placed in the cavity, the optical pulses exhibit excess optical bandwidth beyond the Fourier transform limit. The principle cause of this extra bandwidth is self-phase modulation of the optical pulses induced by gain and absorption saturation. The self-phase modulation process which is important for semiconductor lasers is outlined in Fig. 6. When a semiconductor amplifier produces an optical pulse with an energy approaching the saturation energy of the amplifier, the gain of the amplifier and thus the carrier density is depleted. Index of refraction changes are coupled to gain changes through the linewidth enhancement factor $\alpha$. The drop in carrier density causes an increase in the index of refraction which in turn phase modulates the optical pulse. In a semiconductor laser amplifier, gain saturation causes a drop in the instantaneous frequency over the duration of the pulse. When a pulse propagates through a saturable absorber, the carrier density increase results in an instantaneous frequency rise in the leading edge of the optical pulse. The effects of self-phase modulation can in general be reduced by decreasing the output power and therefore the amount of self-phase modulation in the device. Note that the chirp that results from mode-locked semiconductor lasers is in general not linear over the central portion
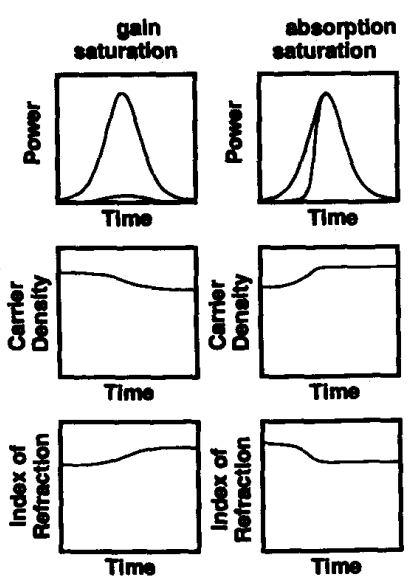

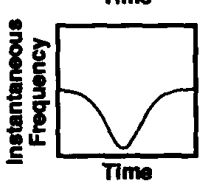

(a)

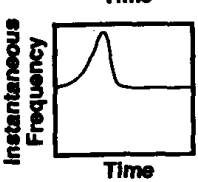

(b)
Fig. 6. Self-phase modulation effects in mode-locked semiconductor lasers. Self-phase modulation due to (a) gain saturation and (b) absorption saturation.

of the pulse. Linear chirp is important because pulses that are linearly chirped lend themselves well to pulse compression techniques. It has been found experimentally that the chirp from both actively and passively modelocked external-cavity lasers has been linear enough to accomplish pulse compression with a modified grating pair compressor [25], [26]. Fig. 7 shows the results of pulse 
compression of a pulse from a two-segment GaAs-AlGaAs passively mode-locked semiconductor laser [20] with a $2 \mathrm{GHz}$ repetition rate. The input pulsewidth is 3.5 ps and a 0.5 ps pulsewidth is produced after compression [27]. For some applications such as optical soliton generation, it is required to have Fourier transform-limited pulses. In external-cavity lasers, it is possible to achieve near transform-limited pulses by using a bandwidth-limiting element such as a diffraction grating in the external cavity. The bandwidth-limiting element filters out the excess bandwidth created by self-phase modulation on each pass at the expense of a wider optical pulsewidth. Fig. 8 shows the results of using a collimated $2 \mathrm{~mm}$ beam on an 830 grooves per millimeter diffraction grating for the device of Fig. 2(b). The pulsewidth from the device increased from $1.4 \mathrm{ps}$ to $7.5 \mathrm{ps}$, but the time-bandwidth product was decreased from 0.48 to 0.30 which is close to the theoretical value of 0.314 for a hyperbolic secant squared pulse shape. Bragg reflectors can also be incorporated into monolithic cavity devices in a similar manner to reduce the optical bandwidth [21]. The bandwidth from gain-switched devices has also been reduced with spectral filters placed outside the cavity for applications in soliton pulse generation [28].

\section{Pulse Energy}

The energy from a mode-locked laser is limited by the saturation energy of the gain and saturable absorber regions. In passively and hybridly mode-locked devices, the output pulse energy is typically near the saturation energy of the gain segment. The pulses from mode-locked semiconductor lasers can be further amplified with external semiconductor or erbium-doped fiber amplifiers. The saturation energy for semiconductor laser amplifiers may range from $1 \mathrm{pJ}$ in a tightly confined index-guided laser up to $20 \mathrm{pJ}$ for lightly confined gain guided edge emitting structures. Erbium-doped fiber amplifiers are limited by the saturation power instead of the saturation energy due to the long lifetime of the gain medium. Average power levels of $10 \mathrm{~mW}$ are obtainable. Vertical-cavity surfaceemitting laser structures, which have much higher saturation energies due to their large mode cross section areas, have been shown to give pulse energies as large as $300 \mathrm{pJ}$ [11].

\section{Timing Jitter and Intensity Noise}

The timing jitter of mode-locked semiconductor lasers is an important parameter since jitter can increase the effective optical pulsewidth for most applications. Absolute timing jitter is the amount of jitter which is contributed by the modulating electrical source together with the mode-locked laser. Residual timing jitter is a measure of the jitter contributed by the mode-locked laser alone, independent of the electrical modulation source. The residual timing jitter from the mode-locked structures shown in Fig. 1 was measured using the phase detector technique [29] and the resulting comparison is shown in Fig. 9. The rms timing jitter was calculated from the data in Fig. 9

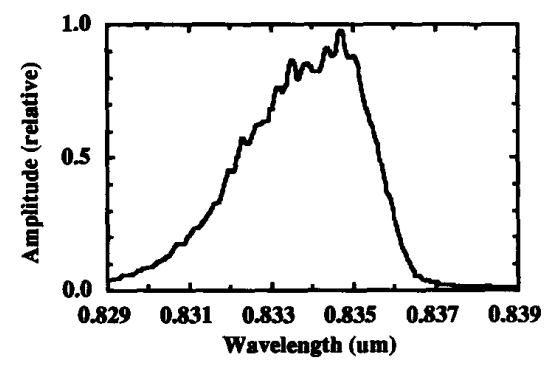

(a)

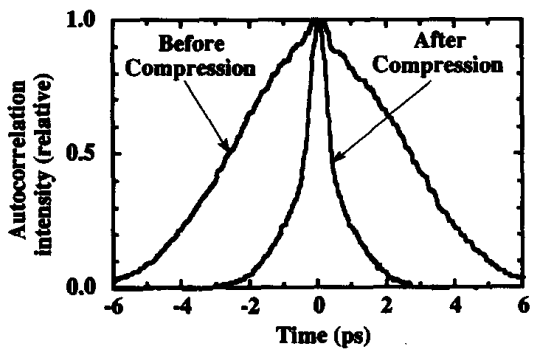

(b)

Fig. 7. Pulse compression of a passively mode-locked two-segment laser as in Fig. 2(c). (a) Optical spectrum before compression. (b) Autocorrelation traces taken before and after pulse compression in a modified grating pair compressor.

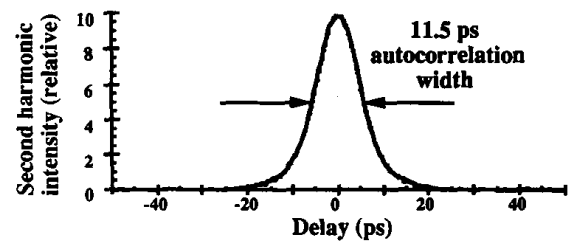

(a)

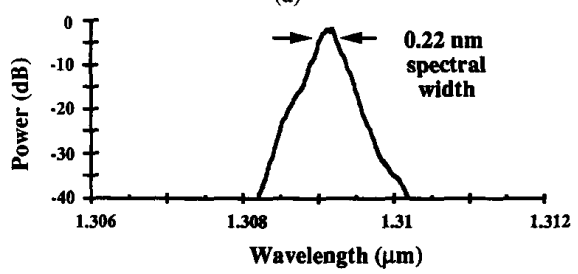

(b)

Fig. 8. (a) Autocorrelation trace and (b) optical spectrum for a two-segment actively mode-locked laser as in Fig. 2(b) with mirror replaced by an 830 grooves per millimeter diffraction grating.

and is listed in Table II. The low frequency intensity noise is also presented. The comparison of mode-locked devices is done with devices from the same GaAs quantumwell wafer and is done at a common repetition rate of $\mathbf{5 . 5}$ $\mathrm{GHz}$ in all cases. Active mode locking of a single-section external cavity laser gave the lowest root mean square residual and absolute timing jitter levels of less than 0.06 ps and $0.25 \mathrm{ps}(150 \mathrm{~Hz}-50 \mathrm{MHz})$ respectively, with a 12 ps pulsewidth. This represents the lowest level of timing jitter measured in any laser system. Such low levels of timing jitter are possible because the mode-locked laser adds very little amplitude and phase noise to that of the electrical modulation source driving the mode locking. Active mode locking of the two-segment external cavity 


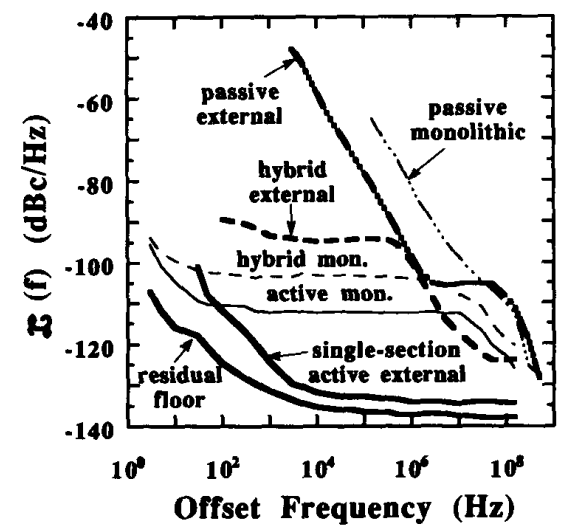

Fig. 9. Residual single side-band phase noise $\mathscr{L}(f)$ comparison for monolithic and external cavity multisection mode-locked semiconductor lasers using active, passive, and hybrid mode-locking techniques. All devices are from the same GaAs-AlGaAs quantum-well wafer.

TABLE II

Comparison of TIMING JitTer and INTENSITY NOISE Levels

\begin{tabular}{llcc}
$\begin{array}{c}\text { Mode- } \\
\text { Locking } \\
\text { Technique }\end{array}$ & \multicolumn{1}{c}{$\begin{array}{c}\text { Absolute rms } \\
\text { Timing Jitter } \\
150 \mathrm{~Hz}-50 \mathrm{MHz}\end{array}$} & $\begin{array}{c}\text { Residual rms } \\
\text { Timing Jitter } \\
150 \mathrm{HZ}-50 \mathrm{MHz}\end{array}$ & $\begin{array}{c}\text { Relative } \\
\text { Intensity Noise } \\
\text { @ } 100 \mathrm{MHz}\end{array}$ \\
\hline $\begin{array}{c}\text { Active Monolithic } \\
\text { Two-Segment }\end{array}$ & $600 \mathrm{fs}$ & $530 \mathrm{fs}$ & $-122 \mathrm{~dB} / \mathrm{Hz}$ \\
$\begin{array}{c}\text { Passive Monolithic } \\
\text { Two-Segment }\end{array}$ & $\begin{array}{c}12.5 \mathrm{ps} \\
(150 \mathrm{kHz}-50 \mathrm{MHz})\end{array}$ & $\begin{array}{c}12.5 \mathrm{ps} \\
(150 \mathrm{kHz}-50 \mathrm{MHz})\end{array}$ & $-116 \mathrm{~dB} / \mathrm{Hz}$ \\
$\begin{array}{c}\text { Hybrid Monolithic } \\
\text { Three-Segment }\end{array}$ & $1200 \mathrm{fs}$ & $1130 \mathrm{fs}$ & $-109 \mathrm{~dB} / \mathrm{Hz}$ \\
$\begin{array}{c}\text { Active External } \\
\text { Single-Segment }\end{array}$ & $240 \mathrm{fs}$ & $65 \mathrm{fs}$ & $<-126 \mathrm{~dB} / \mathrm{Hz}$ \\
$\begin{array}{c}\text { Passive External } \\
\text { Two-Segment }\end{array}$ & $12.2 \mathrm{ps}$ & $\begin{array}{c}612.2 \mathrm{ps} \\
(1.5 \mathrm{kHz}-50 \mathrm{MHz})\end{array}$ & $-103 \mathrm{~dB} / \mathrm{Hz}$ \\
Hybrid External & $1060 \mathrm{fs}$ & $980 \mathrm{fs}$ & $-105 \mathrm{~dB} / \mathrm{Hz}$ \\
Two-Segment & & & \\
\hline
\end{tabular}

laser in [7] resulted in an absolute timing jitter of $0.5 \mathrm{ps}$ $(150 \mathrm{~Hz}-500 \mathrm{MHz})$ with a pulsewidth of $2.2 \mathrm{ps}$. Hybrid mode locking gave somewhat higher levels of timing jitter [e.g., 0.6-1 ps rms jitter $(150 \mathrm{~Hz}-50 \mathrm{MHz})$ ] than those using active mode locking. Passive mode locking has the highest level of timing jitter due to the absence of a highstability driving source. Monolithic cavity mode-locked lasers with all-active waveguides tend to have higher phase noise levels than their external cavity counterparts at the same repetition rate. Monolithic cavity devices which incorporate large sections of low-loss passive waveguide should give comparable timing jitter to their external cavity counterparts. Gain-switched and $Q$-switched lasers give somewhat higher timing jitter levels than mode-locked lasers. The increased timing jitter is due to the fact that each gain-switched and $Q$-switched laser pulse starts each time from amplified spontaneous emission which adds a randomness to the output pulse timing [30].

Spontaneous emission causes gain, index of refraction, and photon density variations. Index of refraction noise can directly lead to timing jitter by introducing a randomness in the round-trip time. Gain and photon density fluc- tuations can also lead to timing jitter through an amplitude to timing jitter conversion process. The amplitude to timing jitter process occurs because variations in the gain and saturable absorber saturation levels lead to variations in the round-trip time. In passive mode locking, spontaneous emission frequency modulates the pulse stream resulting in the $20 \mathrm{~dB}$ per octave rolloff with offset frequency. In active and hybrid mode locking, spontaneous emission phase modulates the pulse stream since active gain modulation limits the peak phase excursions. The level of spontaneous emission noise and thus timing jitter increases with the number of longitudinal modes present and with the gain level in the semiconductor laser amplifier.

Mode-locked lasers are also susceptible to large amplitude and timing jitter instabilities if the low frequency resonance response is not highly damped [31]. This instability can be seen in actively mode-locked lasers when the modulation frequency is tuned to a higher frequency than the natural resonance frequency of the cavity [32]. Passively and hybridly mode-locked lasers are particularly susceptible to this instability because the positive feedback of the saturable absorber reduces the damping of the 


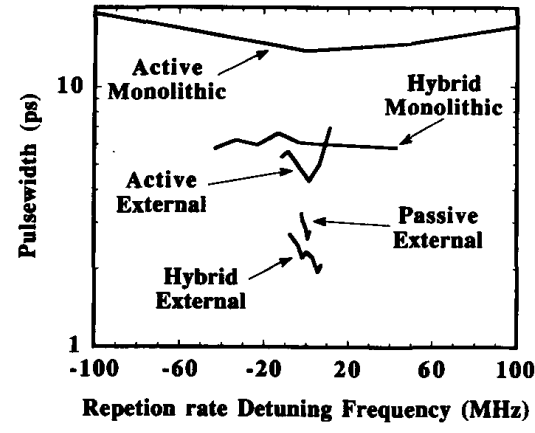

Fig. 10. Pulsewidth versus detuning of the repetition rate. The monolithic cavity device has a center repetition rate of $13 \mathrm{GHz}$ and the external cavity device has a center repetition rate of $6.1 \mathrm{GHz}$. The device structures were that of Fig. 1(a) and (b) with a GaAs bulk active region and $16 \mu \mathrm{m}$ modulation and saturable absorber segment lengths.

low frequency resonance peak. Lasers with saturable absorbers of very high unsaturated loss are more susceptible to self-pulsations. The instability is also found to increase with the repetition rate of the laser.

\section{E. Repetition Rate}

For repetition rates in the hundreds of $\mathrm{MHz}$ to $20 \mathrm{GHz}$ range, external-cavity mode-locked lasers work well. The upper limit on the repetition rate of external cavity lasers is imposed by the finite length of the external cavity optical elements. Monolithic cavity devices have been shown to operate at repetition rates between 5 and $350 \mathrm{GHz}$. The lower repetition rate is limited by fabrication technology limits for monolithic devices approaching $1 \mathrm{~cm}$ in length. The upper repetition rate limit is reached with passive mode-locking techniques and is limited by the saturable absorber recovery time constant. In mode-locked lasers, the repetition period is limited to be near the round-trip time in the device. For a fixed cavity length, mode-locked lasers can be slightly tuned from this resonance with minimal degradation in device performance. The pulsewidth performance versus repetition rate of actively and hybridly mode-locked monolithic and external cavity lasers is compared in Fig. 10. The monolithic device is a 2.75 $\mathrm{mm}$ long bulk GaAs active region device [20] operating at $13 \mathrm{GHz}$. The external-cavity device is $500 \mu \mathrm{m}$ long, operates at $6.5 \mathrm{GHz}$ repetition rate, and comes from the same wafer as the monolithic cavity device. The plotted tuning range limits represent the points at which the modelocked lasers exhibit significant phase or amplitude instabilities as measured in an electrical spectrum analyzer with a photodiode input. Monolithic cavity devices show a greater tunability that external cavity devices. This is presumably due to the lower cavity $Q$ of the monolithic cavity device with all-active waveguide and the fact that the carrier density and thus the index of refraction can self-adjust to bring the laser back into resonance with the modulation signal. Mode-locked lasers which incorporate distributed Bragg reflectors in the cavity have shown a larger tuning range due to the frequency dependence of the effective reflection point [33]. Passively mode-locked semiconductor lasers can be tuned electronically with the incorporation of a repetition rate tuning element as is shown in Fig. 2(e). Repetition rate tuning segments work by changing the gain saturation level in the device which in turn changes the effective round-trip time in the device. This electronic tuning of the repetition rate is important because it provides a convenient way of phase locking the device to a lower frequency standard to reduce the timing jitter [19]. The photocurrent from the saturable absorber also provides a convenient electrical input for the phaselocking circuitry.

\section{Optimization of Multisegment Designs}

The multisegment lasers discussed in the first section have given short optical pulses in a simple structure where gain segments, saturable absorber segments, modulation segments and tuning segments are fabricated together in a single integration process. In this section, a theoretical analysis is performed to understand how to optimize these structures. The variables that the designer has under his control are:

1) active region composition, waveguide dimensions, and optical confinement factor,

2) segment length,

3) electrical parasitics of the segments (capacitance and resistance), and

4) electrical drive signal in active and hybrid mode locking.

With these variables as free design parameters, this section will outline the tradeoffs involved in getting the best device performance. Items 3) and 4) have been discussed in Section II. Minimization of electrical parasitics is especially important for actively mode-locked lasers and gain-switched lasers, in which narrow electrical pulsewidths are necessary to create narrow optical pulses. Passively mode-locked lasers can tolerate larger electrical parasitics since saturable absorption is the dominant pulseshortening mechanism.

\section{A. Simplified Model for Multisegment Mode-Locked Lasers}

The configuration used to analyze passively modelocked lasers is shown in Fig. 11. The model shows a two-segment laser in which the first segment is the gain section and the second segment is the saturable absorber. This model is analyzed with the rate equation set similar to that outlined by Agrawal and Olsson [34], [24]:

$$
\begin{aligned}
\frac{\partial g}{\partial t} & =\frac{\left(g_{o}-g\right)}{t_{c}}-\frac{g P}{E_{\text {sat }}} \\
P_{\text {out }}(\tau) & =\frac{P_{\text {in }}(\tau)}{1-\left(1-1 / G_{o}\right) \exp \left(-U_{\text {in }}(\tau) / E_{\text {sat }}\right)}
\end{aligned}
$$




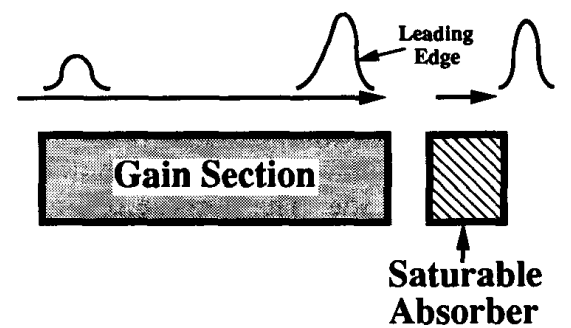

Fig. 11. The two-segment laser model for passive mode locking. The pulse is analyzed for each trip around the semiconductor laser diode.

$$
\phi_{\text {out }}(\tau)=\phi_{\text {in }}(\tau)+\frac{\alpha}{2} \ln \left[1-\left(\frac{1}{G_{o}}\right) \exp \left(\frac{-U_{\text {in }}(\tau)}{E_{\text {sat }}}\right)\right]
$$

$$
\begin{aligned}
U_{\text {in }}(\tau) & =\int_{-\infty}^{\tau} P_{\text {in }}(\tau)^{\prime} d \tau^{\prime} \\
A_{\text {out }}(\tau) & =F^{-1}\left[\tilde{A}_{\text {in }}(\omega) \exp \left(\frac{g L / 2}{1+i t_{2} \omega}\right)\right] .
\end{aligned}
$$

$P_{\text {in }}$ and $P_{\text {out }}$ are the input and output power from a laser segment, $\phi_{\text {in }}$ and $\phi_{\text {out }}$ are the input and output phase from a laser segment, $\tau$ is the time measured with respect to the middle of the optical pulse, $g$ is the gain per unit length, $L$ is the segment length, $g_{o}$ is the maximum available gain for given pump rate, $G_{o}=\exp \left(g_{0} L\right)$ is the unsaturated gain of the segment, $\alpha$ is the linewidth enhancement factor, $P$ is the average power level across a segment, $t$ is the absolute time, $t_{c}$ is the carrier lifetime, $t_{2}$ is an optical bandwidth parameter, $\omega$ is the optical frequency measured with respect to the peak of the gain, $\tilde{A}_{\text {in }}(\omega)$ is the Fourier transform of the input pulse electric field envelope, $\beta_{2}$ is the material dispersion parameter, and $F^{-1}$ is the inverse Fourier transform. Equations (3)(5) relate the input and output pulse waveforms of a laser segment without the bandwidth effects of the laser and assuming that the pulsewidth is much shorter than $t_{c}$. Equation (6) relates the input and output pulse waveforms for a laser segment for the case of no gain saturation effects and includes the magnitude and phase response of gain medium. The magnitude and phase response is chosen to have a Lorentzian shape and does not use Agrawal's assumption of a parabolic gain and phase response [24]. Equation (2) is used to keep track of the time dependence of the gain value in each laser segment. The two-segment device is analyzed by breaking up the model of Fig. 11 into small segments and applying (2)-(6) to each segment. The solution method for the following sections is the two-step method outlined by Agrawal [35] in which first the nonlinearity is calculated [(3)-(5)] and then the bandwidth and the dispersion effects are calculated [(6)]. Since (3)-(6) represent analytical solutions to the input output relationships of a laser segment, fewer segments are needed for accurate simulations. Mode-locked lasers with counter-propagating waves can be simulated with a partial-integration method, where the laser is broken up into several segments which are solved analytically [36].

In this section, results will be first presented for the simplest model in which the linewidth enhancement factor is set to zero and an infinite gain bandwidth is assumed $\left(t_{2}=0\right)$. This simplified model shows more clearly how to maximize the pulse shortening mechanism in passively mode-locked lasers. The effects of self-phase modulation and the associated frequency chirp are then introduced. Finally, the gain and phase response of the amplifier is added to the model in Section V. The interplay of pulse shaping mechanisms, self-phase modulation, and gainbandwidth effects that ultimately limits the minimum achievable pulsewidth is then discussed.

\section{B. Pulse Shortening with Saturable Absorbers}

In this discussion, the process of passive mode locking will be described in terms of how much shorter the optical pulsewidth becomes on each pass around the laser cavity. The optimization of a passively mode-locked laser for shortest pulsewidth is equivalent to the search for the condition of maximum pulse shaping per pass [42]. Fig. 12(a) shows the pulsewidth reduction per pass and Fig. 12(b) shows the net energy gain per pass for the two-segment laser model of Fig. 11 as a function of the input energy divided by the saturation energy of the amplifier. Gain bandwidth and self-phase modulation effects are not included in this simulation. The input pulse shape has a hyperbolic secant squared shape and the full width at half of maximum (FWHM) is used for pulsewidth measurement. At low input energies, the amplifier cannot saturate the absorber leading to a small net gain through the gain-absorber cascade. As the input energy is increased, the absorber starts to saturate and the pulsewidth becomes shortened. As the amplifier gain becomes more and more saturated with increasing input energy, the amplifier widens the input pulse. There exists an input power at which the system experiences the largest pulse shaping per pass. The input energy for maximum pulse shortening per pass occurs at a similar energy to that of the maximum net gain. The maximum net energy gain for these simulations is 25 , corresponding to a typical value for an external cavity laser. The ratio of saturation energies between the gain segment and the absorber segment is an important number in maximizing the pulse shortening per pass. Fig. 12 is plotted for a ratio of saturation energies of 3 and gives a corresponding minimum net pulse shortening ratio of 0.82 . The input energy for maximum pulse shortening is lowered and the maximum pulse shaping per pass is increased for higher ratios of saturation energies because the saturable absorber is more easily saturated. Quantumwell devices generally have a higher saturation energy ratio and thus have a larger pulse shortening ability as compared to bulk active region devices.

Fig. 13(a) is a plot of the maximum pulse shortening per pass in a gain-absorber cascade as a function of the unsaturated transmission value through the saturable ab- 


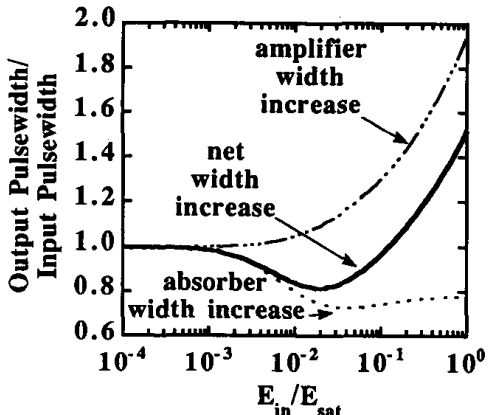

(a)

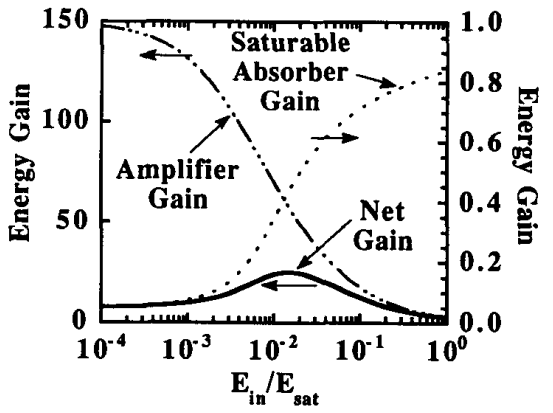

(b)

Fig. 12. (a) Pulse shortening per pass and (b) net energy gain per pass through the gain-absorber cascade laser model of Fig. 11 as a function of the input energy divided by the saturation energy of the amplifier. The net energy gain through the device is 25 . The unsaturated gain of the amplifier is 150 and the unsaturated transmission of the absorber is 0.05 . The ratio of saturation energies between the gain segment and saturable absorber segment is 3 .

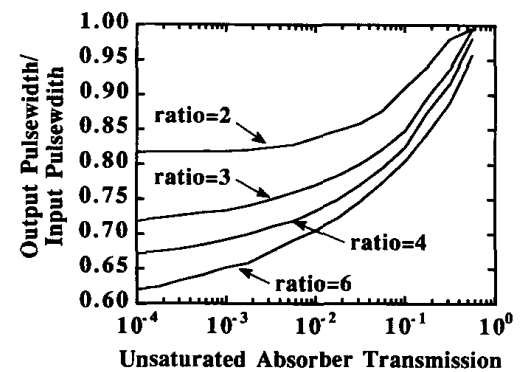

(a)

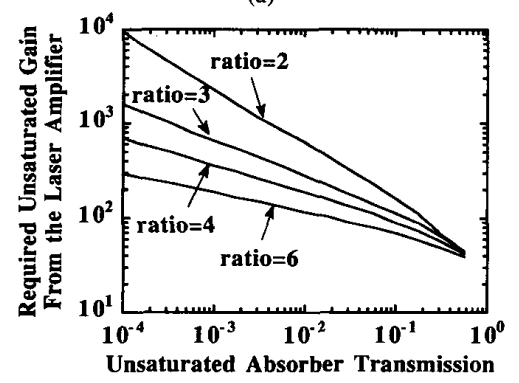

(b)

Fig. 13. (a) Maximum pulse shortening per pass and (b) required unsaturated energy gain from the amplifier for a single pass through the gainabsorber cascade laser model of Fig. 11 as a function of the unsaturated transmission level through the saturable absorber. The simulation is done for four values of the ratio of saturation energies between the gain and absorber segment. The net gain for the gain-absorber cascade is 25 in all cases. sorber. The figure shows various values of the ratio of saturation energy between the gain and absorber segment and is done for a net energy gain of 25 through the device. Fig. 13(b) shows the unsaturated gain value which is required to achieve a saturated net energy gain of 25 through the gain-absorber cascade. The purpose of Fig. 13 is to find the optimal saturable absorber length and gain length for maximization of the pulse shortening per pass in a passively mode-locked semiconductor laser. Fig. 13(a) clearly shows that increasing the ratio of saturation energies is very important to obtain stronger pulse shortening effects. Increasing the ratio of saturation energies also reduces the unsaturated gain requirements for the laser amplifier as is shown in Fig. 13(b). As the unsaturated absorber transmission value decreases, the pulse shortening per pass increases monotonically. For small values of unsaturated absorber transmission, the pulse shortening per pass becomes a weak function of the absorber length. As the unsaturated absorber transmission decreases, the required unsaturated gain from the amplifier increases very rapidly. At some point, the required gain from the amplifier will be unachievable due to too large of required carrier density values for a practical device, or from excessive spontaneous emission noise amplification [37]. Unsaturated energy gain values over 1000 are difficult to achieve due to gain saturation caused by amplified spontaneous emission. Devices with large values of unsaturated absorber transmission are also prone to self-pulsations at the relaxation oscillation frequency of the device. For example, a $40 \mathrm{GHz}$ monolithic cavity mode-locked laser with an $80 \mu \mathrm{m}$ long saturable absorber would only self-pulsate at a $1-2 \mathrm{GHz}$ rate. However, wide regions of stable mode locking existed when the absorber length was reduced to $40 \mu \mathrm{m}$. The maximum absorber length that does not induce self-pulsation effects decreases with increasing repetition rate.

The best compromise is to choose a value of unsaturated absorber transmission located where the pulsewidth shortening function is slowly changing but not at the maximum value of pulse shortening. For Fig. 13(a) this would occur for an unsaturated absorber transmission between approximately 0.01 and 0.2 . This represents a range of saturable absorber lengths which possess the largest pulse shortening per pass without requiring too large of unsaturated gain from the laser amplifier and reducing the susceptibility to self-pulsation effects in the mode-locked laser.

\section{Colliding Pulse Effects}

Passive mode locking can be improved with colliding pulse effects in saturable absorbers. The pulse shortening characteristics for the three different saturable absorbers configurations shown in Fig. 14 are studied [27]. Configuration $\mathrm{A}$ is a unidirectional mode-locked laser (UM) in which there are no pulse collisions in the saturable absorber. Configuration B is a self-colliding pulse modelocking configuration (SCPM) in which the optical pulse collides with itself in the saturable absorber. Configura- 


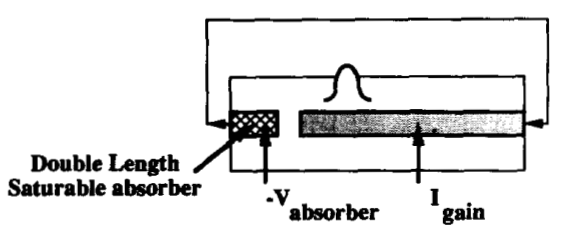

Unidirectional Configuration

(a)

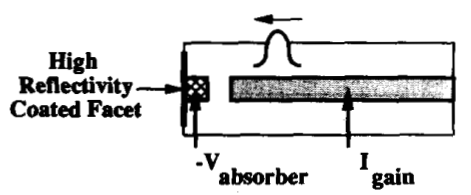

Self-Colliding Pulse Configuration

(b)

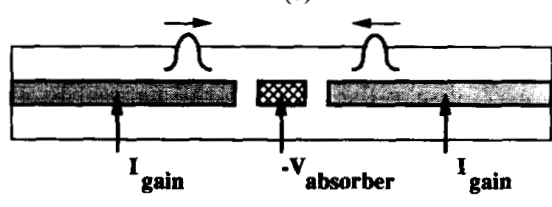

Colliding Pulse Configuration

(c)

Fig. 14. Comparison of saturable absorber configurations.

tion $\mathrm{C}$ is a colliding pulse mode-locking (CPM) configuration in which oppositely directed pulses collide in the saturable absorber.

The CPM and SCPM configurations are nearly the same in terms of saturable absorber performance [38]. The effectiveness of the saturable absorber is enhanced in the SCPM configuration when compared to the UM configuration. Absorbers in the SCPM configuration can be half as long as those in the UM configuration for the same unsaturated attenuation value. Half as many carriers are needed to reach transparency, reducing the energy needed to bleach the saturable absorber by a factor of two. In the CPM and SCPM configurations, there is an additional enhancement due to the interaction of counter-propagating optical pulses in the saturable absorber. The counterpropagating pulses form standing waves in the saturable absorber. At the peak of the standing wave, the carrier generation rate is high, and at the nulls, the carrier generation rate is low. This leads to a carrier density absorption grating. Since the carrier density does not have to be raised to transparency everywhere in the saturable absorber to reach a low loss state, the saturable absorber bleaches with a lower pulse energy than would be necessary if the standing waves did not exist.

The effects of pulse collision in saturable absorbers has been studied in dye lasers [39]-[42]. The optical standing waves form an absorption grating which couples the forward and reverse traveling waves. The equations for coherent pulse interaction [42] can be found from the coupling coefficients of a conductivity grating. The absorption grating is assumed to be sinusoidal.

$$
\alpha=\alpha_{A}+\alpha_{B} \cos 2 k z
$$

The coupled equations for a short segment of length $L$ are:

$$
\begin{aligned}
h_{i} & \equiv \int_{0}^{L}-\alpha_{i} d x \cong-\alpha_{i} L \quad(i=A, B) \\
\frac{d h_{A}}{d t} & =\frac{-\alpha_{0} L-h_{A}}{\tau_{c}}-\frac{\left(A_{\text {in }}^{+}\right)^{2}+\left(A_{\text {in }}^{-}\right)^{2}}{E_{\text {sat }}} h_{A}+\frac{\left(A_{\text {in }}^{+}\right)\left(A_{\text {in }}^{-}\right)}{E_{\text {sat }}} h_{B}
\end{aligned}
$$

$\frac{d h_{B}}{d t}=-\frac{h_{B}}{\tau_{c}}-\frac{\left(A_{\text {in }}^{+}\right)^{2}+\left(A_{\text {in }}^{-}\right)^{2}}{E_{\text {sat }}} h_{B}+2 \frac{\left(A_{\text {in }}^{+}\right)\left(A_{\text {in }}^{-}\right)}{E_{\text {sat }}} h_{A}$

$A_{\text {out }}^{+}=\left(1+\frac{h_{A}}{2}\right) A_{\text {in }}^{+}-\frac{h_{B}}{4} A_{\text {in }}^{-}$

$A_{\text {out }}^{-}=\left(1+\frac{h_{A}}{2}\right) A_{\text {in }}^{-}-\frac{h_{B}}{4} A_{\text {in }}^{+}$.

$A^{+}$and $A^{-}$are the forward and reverse traveling field values. The unsaturated transmission of the segment is exp $\left(-\alpha_{0} L\right)$. The total absorption integrated over the segment length is $h_{A}$ for the dc absorption component and $h_{B}$ for the sinusoidal absorption component due to the carrier grating. The net gain $h$ is found by integrating the gain over the length of a computational segment [34]. The value of $h$ is then negative for absorber segments.

The theoretical performance of a saturable absorber with a typical loss found in mode-locked dye lasers is shown in Fig. 15(a). The saturation energy is a factor of two smaller for incoherent SCPM over the UM configuration for low energy pulses [39]. The coherent SCPM case (with grating) has improved pulse shaping per pass and less loss per pass as compared to the incoherent SCPM case (no grating) for low input energy pulses. For high energy input pulses, the standing wave grating diminishes during the pulse propagation until the absorber becomes almost uniformly bleached. The total energy lost is nearly the same for incoherent and coherent SCPM, but the energy is lost later in the optical pulse for coherent SCPM. The net effect for coherent SCPM is to reduce the energy for optimum pulse shaping per pass and reduce the maximum pulse shaping per pass.

The theoretical performance of a saturable absorber with a typical loss found in mode-locked semiconductor lasers is shown in Fig. 15(b). The unsaturated absorption is much higher in semiconductor lasers compared to dye lasers due to the higher gain per pass in the amplifier section. The effect of the carrier grating for semiconductor lasers is smaller than for the dye laser case. The large initial loss in semiconductor saturable absorbers causes reduced standing-wave effects. Only the section nearest the mirror receives the full benefit of coherent SCPM. The coherent SCPM interaction increases the pulse shaping per pass and reduces the energy for bleaching but at reduced levels compared to the dye laser case. An additional effect in mode-locked semiconductor lasers is the rapid diffusion of the carriers that tends to average out the carrier density grating for wide pulsewidths and further reduces the effects of coherent SCPM.

The effects of colliding pulses were studied experimen- 


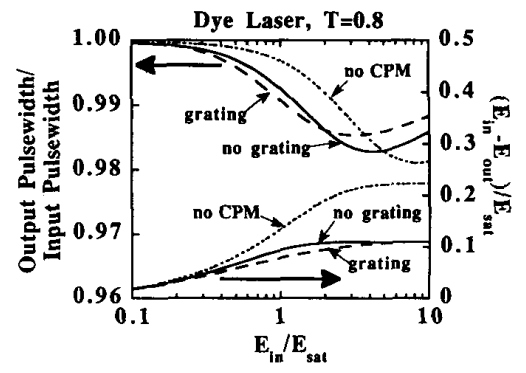

(a)

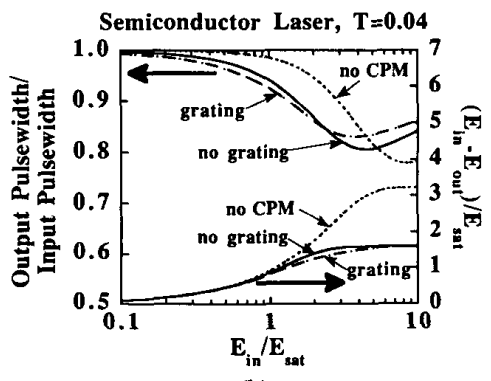

(b)

Fig. 15. Calculated pulse shaping per pass and normalized energy loss per pass, $\left(E_{\text {in }}-E_{\text {out }}\right) / E_{\text {sat }}$, for a single pass through the saturable absorber configurations of Fig. 14. Part a simulates the typical case for a passively mode-locked dye laser and part $b$ simulates the typical case for a passively mode-locked semiconductor laser.

tally by varying the facet reflectivity to change the depth of the optical standing waves. The results are shown in Fig. 16. It is seen that a facet field reflectivity reduction from 80 to $55 \%$ resulted in no increase in pulsewidth. Even for field reflectivities as small as $14 \%$ (power reflectivity of $2 \%$ ), in which the colliding pulse effect is small, short pulses were produced. The small increase in pulse width for very low mirror reflectivity could also be attributed to the increased self-phase modulation caused by the need for increased gain in the laser.

The incoherent colliding pulse effect in mode-locked semiconductor lasers is important because it allows a passively mode-locked laser to operate at a lower pulse energy for maximum pulse shortening, and therefore reduce the pulse broadening effects of self-phase modulation. We have found that the coherent SCPM improvement in pulse shaping from the carrier grating is small, particularly in semiconductor diode laser mode locking, and that the main effect is from the advantage of incoherent pulse collision.

\section{Active Region Design}

Quantum-well lasers give the largest ratios in saturation energies between the gain and saturable absorber sections and therefore should perform better than bulk active region devices. Experimentally, both quantum-well and bulk active region devices have given excellent results in external and monolithic cavity experiments. To take ad-

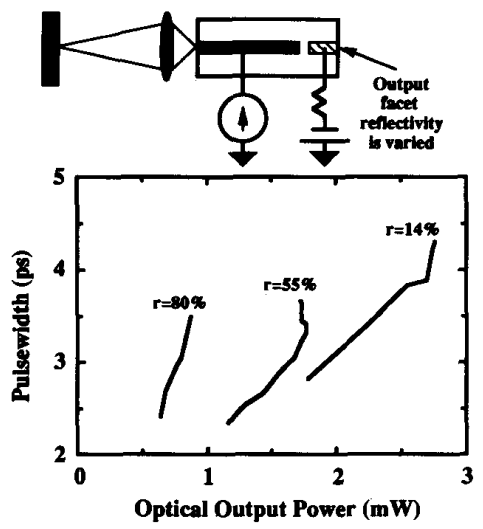

Fig. 16. Experimental pulsewidth versus output power for three different field reflection coefficients on the saturable absorber facet. The repetition rate is $2.5 \mathrm{GHz}$ for all cases.

vantage of colliding pulse effects, the saturable absorber length should be shorter than the optical pulsewidth so that a standing wave can be formed over the entire length of the absorber. Thus, choosing a saturable absorber design with a large optical confinement factor should give better results. It is also important to design the structure so that efficient carrier sweep out can occur in reversebiased absorber segments. Trapping of carriers at heterojunction interfaces or deep quantum wells tend to increase the carrier sweep-out time. Quantum-well devices also give lower levels of self-phase modulation since the linewidth enhancement factor is typically lower than in bulk active region designs.

\section{Limits on the Achievable Pulsewidth}

When an optical pulse travels around the mode-locked laser cavity, there are mechanisms that widen the optical pulse and there are mechanisms that narrow the optical pulse. The final solution to the pulsewidth is obtained when a balance is reached between the widening and shortening mechanisms.

The dominant shortening mechanisms are:

1) saturable absorption (passive or hybrid mode locking)

2) active gain modulation (active or hybrid mode locking).

The dominant widening mechanisms are:

1) the gain response (magnitude and phase versus optical frequency) of the laser,

2) self-phase modulation due to saturable gain, saturable absorption and active gain modulation,

3) saturable gain,

4) dispersion and bandwidth limiting in external cavity optical elements, and

5) spontaneous emission noise.

Saturable absorption is the strongest pulse narrowing 
mechanism since the pulse shaping per pass is constant as the pulsewidth narrows. Pulse narrowing by active gain modulation is very strong for wide optical pulsewidths but becomes weak as the optical pulsewidth becomes narrow in comparison to the active gain modulation electrical pulsewidth. The gain response of the laser amplifier increases the pulsewidth on each pass strongly as the pulsewidth approaches the value of $t_{2}$. The final solution to the pulsewidth is found to be a result of the interplay between pulse narrowing by saturable absorption, excess bandwidth production caused by self-phase modulation, and gain response effects.

\section{A. Self-Phase Modulation}

Self-phase modulation results when optical pulses induce changes in the index of refraction in the material during pulse propagation. The dominant mechanism for self-phase modulation in semiconductor lasers is outlined in Fig. 6. Active gain modulation also causes frequency chirp on the laser pulse. The carrier density rise during the leading edge of the optical pulse in active gain modulation produces a frequency rise similar to that of the saturable absorption induced self-phase modulation of Fig. 6(b). The magnitude of the chirp components is very dependent on the relative magnitude of $\alpha$ in the gain and absorber segments and on the saturation energies in each segment. The chirp would build up with each pass through the laser indefinitely if it were not for the gain and phase response of the laser which ultimately limits these frequency excursions and therefore limits the achievable pulsewidth. Self-phase modulation is the dominant mechanism for excess bandwidth production beyond the Fourier transform limit in mode-locked semiconductor lasers.

\section{B. Laser Amplifier Magnitude and Phase Response}

The magnitude and phase response versus frequency of the semiconductor medium is described by the optical transfer function in (6). The magnitude and phase response of the amplifier are modeled with a Lorentzian line shape. Both the magnitude and phase response of the amplifier can cause pulse widening of a mode-locked laser pulse. The pulsewidth narrowing of active gain modulation and saturable absorption is eventually counteracted by the gain response widening. The amplifier broadens input pulses with excess optical bandwidth much more effectively than pulses with Fourier transform limited spectral width [43].

\section{Limits on the Achievable Pulsewidth}

This section examines how the pulse narrowing effects interact with the important pulse widening effects to reach an equilibrium value of pulsewidth. Fig. 17 shows the pulsewidth versus pass number through the gain-absorber cascade passively mode-locked laser model of Fig. 11 for

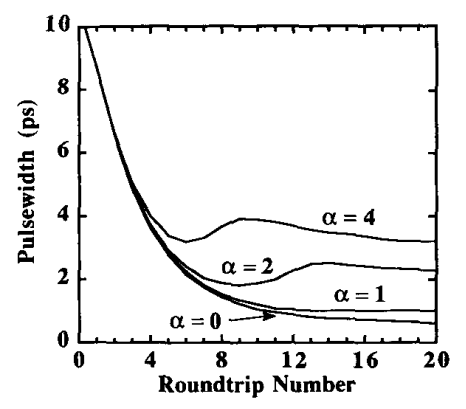

Fig. 17. Pulsewidth versus round-trip number for a two section passively mode-locked laser with increasing values of the linewidth enhancement factor $\alpha$. The gain and phase response of the laser is included for all cases $\left(t_{2}=0.1 \mathrm{ps}\right)$.

increasing values of the linewidth enhancement factor $\alpha$. The bandwidth parameter $t_{2}$ is $0.1 \mathrm{ps}$, and the ratio of saturation energies between the gain and absorber segments is 3. The unsaturated gain of the amplifier is 150 and the unsaturated transmission in the absorber is 0.05 . These parameters have been chosen to simulate the passively mode-locked laser experiment of Fig. 7.

Subpicosecond pulsewidths are easily achieved when self-phase modulation effects are not present in the passively mode-locked laser $(\alpha=0)$. As the magnitude of $\alpha$ is increased, the amount of self-phase modulation is also increased resulting in optical pulses with increasing optical spectral widths and time-bandwidth products. Pulses that are 1-2 ps wide can build up a self-phase modulation induced spectral width equivalent to that found in a transform-limited pulsewidth of a few hundred femtoseconds. The gain and phase response of the laser amplifier broadens the pulsewidth of the large spectral width input pulses much more effectively than transform-limited input pulses of the same pulsewidth. The interaction of selfphase modulation together with the gain and phase response of the laser limit the achievable pulsewidth to a much larger value than would be possible if the self-phase modulation effect were not present. This simulation shows similar final pulsewidth and spectral characteristics to that of Fig. 7 assuming $\alpha$ is in the $2-4$ range. The achievable pulsewidths for multisegment passively and hybridly mode-locked lasers are typically over $1 \mathrm{ps}$ for $t_{2}$ greater than $0.1 \mathrm{ps}$ and $\alpha$ factors greater than 2 .

It is possible to get a smaller $t_{2}$ value by properly spacing the quantized energy levels in a quantum-well laser. Increasing the ratio of saturation energies between the saturable absorber and the gain section increases the pulse shaping per pass and therefore decreases the minimum achievable pulsewidth. The final chirp on the pulse formed in the simulation of Fig. $17(\alpha=4)$ was low in frequency on the leading edge of the optical pulse and high in frequency on the trailing edge of the optical pulse. This frequency up-chirp is consistent with the measured sign of the chirp in the passively mode-locked laser compressed pulse experiment of Fig. 7. In general, the final shape of 
the frequency chirp is highly dependent on the values of $\alpha$ chosen for the saturable absorber and gain sections, the required gain from the semiconductor laser, the loss in the saturable absorber, the active gain modulation induced chirp, and the detuning of the active gain modulation frequency from the natural cavity frequency. The value of $\alpha$ can be adjusted by introducing strain or quantum confinement or by adjusting the material's bandgap relative to the lasing frequency. Values of $\alpha$ from 1 to 20 have been measured. It may be possible to find certain combinations of these parameters which will eliminate or reduce frequency chirp as is suggested by the result in [7]. An alternative solution is to provide the correct phase filtering inside the cavity to counteract self-phase modulation on each pass which has been successful in dye laser systems.

\section{Future Directions ANd Conclusion}

The effects of self-phase modulation at present represent the biggest obstacle in obtaining high power, subpicosecond optical pulses directly from multisegment modelocked lasers. Researchers have initially used the excess optical bandwidth to compress the optical pulses down to subpicosecond widths. The next step is to provide the correct phase filtering inside the cavity to compensate for the self-phase modulation effects and phase dispersion of the laser diode on each pass. There has been work in this area with the use of a Gires-Tournois interferometer in an external cavity [44]. There is promise in applying this to the multi-segment structures which produce narrower initial pulsewidths. Multisegment mode-locked semiconductor lasers can also be combined with the more ideal gain medium in Er-doped fiber amplifiers. The semiconductor can provide fast gain modulation and saturable absorption while the erbium amplifier provides smaller self-phase modulation levels. There are also many other structures which can be constructed which are compatible with the multisegment configurations. The pump-probe measurements show that saturable absorbers are useful as optically controlled optical switches with less than $15 \mathrm{ps}$ recovery time. This should be a useful effect for the design of all-optical demultiplexers, optical sampling oscilloscopes, or in optical logic gates.

In summary, the design and experimental characterization of multisegment short-pulse semiconductor laser structures has been studied. The problem of multiple pulsations in single-segment external cavity lasers have been solved with the use of two and three-segment laser designs. Pulsewidths in the 1-3 ps range are shown to be easily obtainable with optimized actively, passively, and hybridly mode-locked devices in monolithic and externalcavity configurations. Optimization of passively and hybridly mode-locked lasers structures in terms of the free design parameters of the laser was discussed. The ultimately achievable bandwidth is shown to be limited by the interaction of self-phase modulation and the gain response in the semiconductor laser.

\section{ACKNOWLEDGMENT}

The authors thank R. Thornton at the Xerox Palo Alto Research Center for providing $0.85 \mu \mathrm{m}$ devices, R. T. Huang at Rockwell International for providing $1.3 \mu \mathrm{m}$ devices, and T. Tanbun-Ek, D. Coblentz, and R. Logan for providing $1.55 \mu \mathrm{m}$ material. The authors also appreciate discussions with J. Heritage, P. Humphrey, W. Jiang, R. Nagarajan, and T. Reynolds.

\section{REFERENCES}

[1] R. S. Tucker, G. Eisenstein, and S. K. Korotky, "Optical time division multiplexing for very high bit-rate transmission," J. Lightwave Technol., vol. 6, pp. 1737-1749, 1988.

[2] P. A. Andrekson, N. A. Olsson, J. R. Simpson, T. Tanbun-Ek, R. A. Logan, and M. Haner, " $16 \mathrm{Gbit} / \mathrm{s}$ all optical demultiplexing using four-wave mixing," Electron. Lett., vol. 27, pp. 922-924, 1991 .

[3] J. Wiesenfeld, "Electrooptic sampling of high speed devices and integrated circuits," IBM J. Res. Develop., vol. 34, pp. 141-161, 1990.

[4] R. A. Becker, C. E. Woodward, F. J. Leonberger, and R. C. Williamson, "Wide-band electrooptic guided wave analog-to-digital converters," Proc. IEEE, vol. 72, pp. 802-819, 1984.

[5] J. E. Bowers, P. A. Morton, A. Mar, and S. W. Corzine, "Actively mode-locked semiconductor lasers, ' IEEE J. Quantum Electron., vol. 25, pp. 1426-1439, 1989.

[6] M. Shell, A. Weber, E. Schol, and D. Bimberg, "Fundamental limits of sub-picosecond pulse generation by active mode-locking of semiconductor lasers: the spectral width and the facet reflectivities," IEEE J. Quantum Electron., vol. 25, pp. 1661-1668, 1991.

[7] A. Mar, D. J. Derickson, R. J. Helkey, and J. E. Bowers, "1.4 ps pulses directly generated using a tandem contact actively mode-locked semiconductor lasers," presented at IEEE Lasers Electro-Opt. Soc., 1991 Ann. Meet., San Jose, CA, paper SDL14.1.

[8] J. Werner, H. Melchior, and G. Guekos, "Stable optical picosecond pulses from actively mode-locked twin-section diode lasers," Electron. Lett., vol. 24, pp. 140-141, 1988.

[9] H. A. Haus, "Theory of mode-locking with a slow saturable absorber," IEEE J. Quantum Electron., vol. QE-11, pp. 736-746, 1975.

[10] G. P. Agrawal and N. K. Dutta, Long Wavelength Semiconductor Lasers. New York: Van Nostrand Reinhold, 1986.

[11] W. B. Jiang, R. Mirin, and J. E. Bowers, "Mode-locked vertical cavity surface emitting lasers," Appl. Phys. Lett., vol. 59, pp. 577$579,1992$.

[12] J. P. Van Der Ziel, W. T. Tsang, R. A. Logan, R. M. Mikulyak, and W. M. Augustyniak, "Subpicosecond pulses from passively mode-locked GaAs buried optical guide semiconductor lasers, " Appl. Phys. Lett., vol. 39, pp. 525-527, 1981.

[13] E. L. Portnoi and A. V. Chelnokov, "Characteristics of heterostructure lasers with a saturable absorber fabricated by deep ion implantation," Sov. Tech. Phys. Lett., vol. 15, pp. 432-433, 1989.

[14] P. W. Smith, Y. Silverberg, and D. A. B. Miller, "Mode-locking of semiconductor diode lasers using excitonic nonlinearities," $J$. Opt. Soc. Amer. B, vol. 2, pp. 1228-1235, 1985.

[15] C. Harder, J. S. Smith, K. L. Lau, and A. Yariv, "Passive modelocking of buried heterostnucture lasers with non-uniform current injection," Appl. Phys. Lett., vol. 42, pp. 772-774, 1983.

[16] D. J. Derickson, R. J. Helkey, A. Mar, J. E. Bowers, and R: L. Thornton, "Suppression of multiple pulse formation in external cavity mode-locked semiconductor lasers using intra-waveguide saturable absorbers,' IEEE Photon. Technol. Lett., vol. 4, pp. 333-335, Apr. 1992.

[17] J. R. Karin, D. J. Derickson, R. J. Helkey, J. E. Bowers, and R. L. Thornton, "Field-enhanced GaAs / AlGaAs waveguide saturable absorbers," submitted to the 1992 Ultrafast Phen. VIII Conf., Antibes Juan-les-Pins, France, 1992.

[18] Y. K. Chen, M. C. Wu, T. Tanbun-Ek, R. A. Logan, and M. A. Chin, "Subpicosecond monolithic colliding pulse mode-locked multiple quantum well lasers," Appl. Phys. Lett., vol. 58, pp. 1253$1255,1991$. 
[19] D. J. Derickson, R. J. Helkey, A. Mar, J. G. Wasserbauer, and J. E. Bowers, "Microwave and millimeter wave signal generation using mode-locked semiconductor lasers with intrawaveguide saturable absorbers," presented at IEEE Int. Microwave Theory Tech. Symp., Albuquerque, NM, 1992 paper V-2.

[20] R. L. Thomton, W. J. Mosby, and T. J. Paoli, "Monolithic waveguide coupled cavity lasers and modulators fabricated by impurity induced disordering," J. Lightwave Technol., vol. 6, pp. 786-792, 1988.

[21] P. B. Hansen, G. Raybon, and U. Koren, " $5.5 \mathrm{~mm}$ long InGaAs monolithic extended cavity laser with an integrated Bragg-reflector for active mode-locking," IEEE Photon. Technol. Lett., vol. 4, pp. 215-217, Mar. 1992.

[22] D. J. Derickson, P. A. Morton, J. E. Bowers, and R. L. Thornton "Comparison of timing jitter in external and monolithic cavity modelocked semiconductor lasers,"' Appl. Phys. Lett., vol. 59, pp. 3372 3374,1991 .

[23] H. Temkin, T. Tanbun-Ek, and R. A. Logan, "Strained InGaAs quantum well lasers," Appl. Phys. Lett., vol. 56, pp. 1210-1212, 1990.

[24] G. P. Agrawal, "Effect of gain dispersion on ultrashort pulse propagation in semiconductor laser amplifiers," IEEE J. Quantum Electron., vol. 27, pp. 1843-1849, 1991 .

[25] M. Kuznetsov, J. M. Wiesenfeld, and L. R. Radzihovsky, "Compression of picosecond pulses using a modified grating pai compressor," Opt. Lett., vol. 15, pp. 180-182, 1990.

[26] P. J. Delfyett, L. Florez, N. Stoffel, T. Gmitter, N. Andreadakis, and G. Alphonse, "Generation of femtosecond high-power optical pulses from a hybrid mode-locked semiconductor laser," presented at Opt. Soc. Amer. Ann. Meet., San Jose, CA, 1991, paper MS3.

[27] R. J. Helkey, D. J. Derickson, A. Mar, J. G. Wasserbauer, and J. E. Bowers, "Colliding pulse effects in mode locked semiconducto diode lasers,"' presented at Conf. Lasers Electro-Opt., Anaheim, CA 1992, paper JThB2.

[28] K. Iwatsuki, K. Suzuki, and S. Mishi, "Adiabatic soliton compres sion of gain-switched DFB-LD pulse by distributed fiber Raman amplification," IEEE Photon. Technol. Lett., vol. 3, pp. 1074-1076, 1991.

[29] D. J. Derickson, A. Mar, and J. E. Bowers, "Residual and absolute timing jitter in actively mode-locked semiconductor lasers," Electron. Lett., vol. 26, pp. 2026-2027, 1990.

[30] A. J. Taylor, J. M. Wiesenfeld, G. Eisenstein, and R. S. Tucker "Timing jitter in mode-locked and gain-switched InGaAsP injection lasers," Appl. Phys. Lett., vol. 49, pp. 681-683, 1986.

[31] K. Y. Lau and J. Paslaski, "Condition for short pulse generation in ultrahigh frequency mode-locking of semiconductor lasers," IEEE Photon. Technol. Lett., vol. 3, pp. 974-976, 1991.

[32] A. J. Lowrey, "New time domain model for active mode-locking based on the transmission line laser model," IEEE Proc. J, vol. 136, pp. 264-270, 1989.

[33] P. A. Morton, R. Adar, R. C. Kistler, C. H. Henry, T. Tanbun-Ek, R. A. Logan, D. L. Coblentz, A. M. Sergent, and K. W. Wecht, "Hybrid soliton pulse source using silica waveguide external cavity and Bragg reflector,' Appl. Phys. Lett., vol. 59, pp. 2944-2946, 1991.

[34] G. P. Agrawal and N. A. Olsson, "Self-phase modulation and spectral broadening of optical pulses in semiconductor laser amplifiers," IEEE J. Quantum Electron., vol. 25, pp. 2297-2306, 1989.

[35] G. P. Agrawal, Nonlinear Fiber Optics. San Diego, CA: Academic, 1989.

[36] R. J. Helkey, P. A. Morton, and J. E. Bowers, "Partial integration method for analysis of mode-locked semiconductor lasers," Opt. Lett., vol. 15, pp. 112-114, 1990.

[37] C. H. Lee and P. J. Delfyett, "Limits on amplification of picosecond pulses by using semiconductor laser traveling wave amplifiers," IEEE J. Quantum Electron., vol. 27, pp. 1100-1114, 1991.

[38] D. J. Derickson, R. J. Helkey, A. Mar, J. E. Bowers, T. TanbunEk, D. Coblentz, and R. A. Logan, "Comparison of colliding pulse and self-colliding pulse monolithic cavity mode-locked semiconductor lasers," presented at Opt. Fiber Conf., San Jose, CA, 1992, paper ThB3.

[39] J. Herrmann, F. Wiedner, and B. Wilhelmi, "Theory of passive mode-locking of $\mathrm{CW}$ dye lasers with contacted and non-contacted absorbers," Appl. Phys. B, vol. 26, pp. 197-202, 1981

[40] W. Dietel, "Transient absorber gratings shorten the pulses of a passively mode-locked CW dye laser," Opt. Commun., vol. 43, pp. 6971,1982
[41] D. Kuhlke, W. Rudolph, and B. Wilhelmi, "Influence of transient absorber gratings on the pulse parameters of passively mode-locked cw dye ring lasers," Appl. Phys. Lett., vol. 42, pp. 325-327, 1983.

[42] M. S. Stix and E. P. Ippen, "Pulse sharing in passively mode-locked ring dye lasers," IEEE J. Quantum Electron., vol. QE-19, pp. 520 $525,1983$.

[43] A. E. Siegman, Lasers. Mill Valley, CA: Univ. Sci. Books, 1986.

[44] J. Kuhl, M. Serenyi, and E. O. Gobel, "Bandwidth-limited picosecond pulse generation in an actively mode-locked GaAs laser with intracavity chirp compensation,"' Opt. Lett., vol. 12, pp. 334-336, 1987.

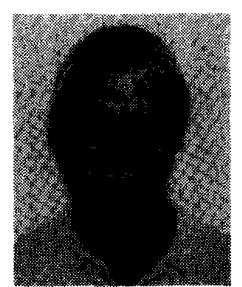

Dennis J. Derickson (M'87) was born in Westbrook, MN, in 1959. He received the B.S, degree in electrical engineering in 1981 from South $\mathrm{Da}$ kota State University, Brookings, and the M.S. degree in electrical engineering in 1982 from the University of Wisconsin, Madison. In 1992 he received the Ph.D. degree in electrical engineering from the University of California, Santa Barbara.

From 1983 to 1988 he was a member of the technical staff at Hewlett-Packard Co., Santa Rosa, CA, working on GaAs monolithic amplifiers, millimeter wave filters, and high-speed optical to electrical convert ers. His current research interests are in the area of optoelectronic device design and fabrication.

Dr. Derickson is a member of the Optical Society of America and the American Physical Society.

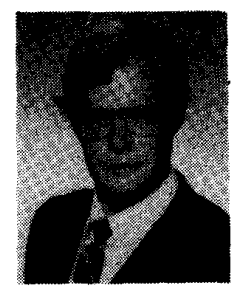

Roger J. Helkey received the B.S. degree in engineering with honors from the California Institute of Technology, Pasadena, in 1982, and the M.S. degree in electrical engineering from the University of California, Santa Barbara, in 1988.

From 1982 to 1984 , he was a design engineer at Trimble Navigation where he was responsible for the design of digital and analog circuits in the development of a Global Positioning System (GPS) receiver. From 1984 to 1986 , he was a member of the Technical Staff at Watkins-Johnson, where he was responsible for microwave component and subsystem design. His current research interests include subpicosecond pulse generation with mode-locked semiconductor diode lasers and theoretical models of semiconductor laser structures. He is presently working toward the Ph.D. degree at the University of California, Santa Barbara.

Alan Mar received the A.B. degree in physics from Occidental College, Los Angeles, CA, in 1985 and the M.S. degree in electrical and computer engineering from the University of California in 1989. His research interests during his studies were in coherent imaging techniques, especially the use of holographic interferometry for deformation analysis and micropositioning of rigid objects

Currently, he is pursuing the Ph.D. degree at the University of California, Santa Barbara, investigating the dynamics of high modulation bandwidth and techniques for short pulse generation from semiconductor lasers.

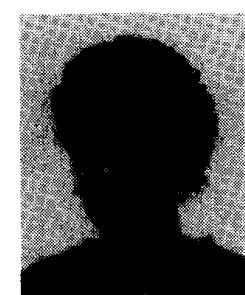

Judy R. Karin (S'86) received the A.B. (magna cum laude) degree in physics in 1983 from Mount Holyoke College, South Hadley, MA and the M.S degree in electrical engineering from the Univer sity of California, Santa Barbara, in 1987.

She is currently working toward the Ph.D. degree at UCSB. From 1983 to 1985 she was with AT\&T Bell Laboratories, Murray Hill, NJ, performing and modeling auto- and cross-correlation measurements on picosecond photoconductors. Her recent research interests include ultrafast dynamics in semiconductor quantum structures and devices. 


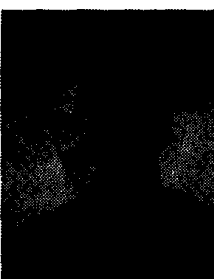

John G. Wasserbauer (S'88) received the B.S. degree in materials science and engineering at Comell University, Ithaca, NY, in 1984. In 1986 he received the D.E.A. degree from École Centrale de Lyon, Écully, France. His studies in France included the growth of GaAs on Si by MOCVD at the Bagneux Laboratory of the Centre Nationale D'Études de Télécommunications.

$\mathrm{He}$ is currently working toward the Ph.D. degree at UCSB. His current research involves the design and fabrication of high-speed long-wave-

length lasers for telecommunications. He is also involved with the design and fabrication of monolithic mode-locking laser structures and other photonic integration processes.

John E. Bowers (S'78-M'81-SM'85), for a photograph and biography, see this issue, p. 2008 . 